\title{
Laurence Guignard, Pascal Raggi, Étienne Thévenin (dir.), Corps et machines à l'âge industriel
}

Rennes, Presses universitaires de Rennes, collection « Histoire », 2011

\section{Anne Marchand}

\section{(2) OpenEdition}

\section{Journals}

Édition électronique

URL : http://journals.openedition.org/travailemploi/5875

DOI : $10.4000 /$ travailemploi.5875

ISSN : 1775-416X

Éditeur

DARES - Ministère du Travail

Édition imprimée

Date de publication : 15 décembre 2012

Pagination : 85-86

ISSN : 0224-4365

Référence électronique

Anne Marchand, «Laurence Guignard, Pascal Raggi, Étienne Thévenin (dir.), Corps et machines à l'âge industriel », Travail et Emploi [En ligne], 132 | octobre-décembre 2012, mis en ligne le 01 décembre

2012, consulté le 22 septembre 2020. URL : http://journals.openedition.org/travailemploi/5875; DOI https://doi.org/10.4000/travailemploi.5875

Ce document a été généré automatiquement le 22 septembre 2020.

(c) Direction de l'animation de la recherche, des études et des statistiques (Dares) 


\title{
Laurence Guignard, Pascal Raggi, Étienne Thévenin (dir.), Corps et machines à l'âge industriel
}

Rennes, Presses universitaires de Rennes, collection « Histoire », 2011

\author{
Anne Marchand
}

\section{RÉFÉRENCE}

Laurence Guignard, Pascal Raggi, Étienne Thévenin (dir.), Corps et machines à l'âge industriel, Rennes, Presses universitaires de Rennes, collection « Histoire », 2011, 321 p.

1 L'invention de la machine à vapeur (1769), celle du moteur à explosion (1862), puis celle du moteur électrique (1887), ont permis le développement de tous types de machines qui se sont imposées bien au-delà du seul monde du travail en investissant celui des loisirs, des transports, de la santé mais également l'espace domestique. Elles sont à l'origine de véritables transformations sociales mais aussi de nouvelles sensations et émotions, de transformations des corps, obligés de s'adapter à un "nouveau milieu ", selon la formule de Georges Friedmann ${ }^{1}$ qui définit ainsi l'ensemble des techniques "qui a transformé et transforme chaque jour les conditions d'existence de l'homme » et « crée, installe, épaissit chaque jour davantage autour de lui ce que nous appellerons le nouveau milieu » (p. 225).

2 Issu d'un colloque organisé en 2010 à Nancy, cet ouvrage se donne pour ambition d'aborder l'histoire des rapports qui s'instaurent entre corps et machines dans le contexte d'industrialisation du xIX siècle. En souhaitant rapprocher deux historiographies encore jeunes - celle du corps, née des travaux de la «nouvelle histoire $»^{2}$ et celle de la santé au travail - il entend dépasser les discours et représentations souvent univoques sur la «machine émancipatrice » versus «broyeuse d'hommes ", pour rendre compte d'une réalité plus complexe et « inclure les machines dans l'histoire d'une anthropologie du corps contemporain » ( $4^{\mathrm{e}}$ de couverture). 
Du baquet de Mesmer au billard électrique (plus tard appelé flipper) en passant par les bombes explosives, la charrue à main de Mathieu de Dombasle, les engins miniers, le physionotrace, le vélocipède, etc., les vingt-trois études qui composent ce recueil abordent une réelle diversité de machines, de domaines d'activité et d'usage, et couvrent une longue période de près de deux cents ans (de la fin du XVIII siècle à la fin $\mathrm{du} \mathrm{xx}^{\mathrm{e}}$ ). Elles sont classées en quatre catégories qui donnent leur titre aux quatre premières parties: "machines de santé ", «machines de mort», "machines de travail» et «machines de loisirs». Une cinquième partie intitulée "régénération et fatigues des corps » s'ouvre à des contributions davantage transversales.

4 À lire cet ouvrage, on est d'abord frappé par sa dimension particulièrement hétéroclite. Sans doute, comme le rappelle Alain Corbin qui signe la préface du livre, parce qu'il n'est pas aisé « de définir avec précision ce qui distingue l'outil et l'appareil de ce qui constitue la machine proprement dite. [...] On comprendra, à la lecture de ce livre foisonnant, la fragilité des limites $»^{3}$ (p.9). Il est donc difficile de rendre compte d'objets, de sources et d'approches si variés. Au lieu d'un survol éclair de l'ensemble, on s'attachera, par-delà les frontières thématiques qui entendaient les distinguer, aux contributions qui justement interrogent les limites entre objet, machine et corps.

Isabelle Renaudet pose ainsi la question des frontières entre l'objet prothèse et le membre mutilé auquel il est censé se substituer, en analysant la trajectoire du docteur Bastos Ansart (1887-1973), pionnier de la chirurgie orthopédique et traumatologique en Espagne, notamment auprès des mutilés de guerre. De conflits en guerres, à travers ses écrits, on aperçoit combien sa représentation du corps du mutilé se transforme. D'abord fervent promoteur de la prothèse, il est persuadé que cette dernière peut parfaitement jouer le rôle d'organe de substitution des membres inférieurs. Au mutilé d'apprendre ensuite, par une rééducation menée par étapes, à maîtriser son nouveau corps jusqu'au moment où la prothèse agira totalement comme une nouvelle jambe. Mais cette approche purement technique trouve ses limites quelques années plus tard: en donnant la parole aux mutilés, Bastos Ansart prend acte de leur rejet de ces objets lourds, froids et sans vie, de leurs difficultés à se réinsérer dans la société. Il s'ouvre alors aux dimensions psychiatriques, psychologiques et sociales de la mutilation, aux souffrances de ses patients et aux discriminations dont ils peuvent être victimes dans l'Espagne franquiste, quand leur corps présente les stigmates de leur engagement républicain. L'objet seul, aussi perfectionné soit-il, ne suffit donc pas à « réparer » un corps amputé.

6 Anne Carol se saisit d'une catégorie particulière de corps, le corps-mort, et suggère une analogie tout à fait originale entre les dispositifs machiniques et le cimetière moderne. Rappelant les changements d'attitude collective face au corps-mort qui apparaissent au $\mathrm{xVIII}^{\mathrm{e}}$ siècle et au début du XIX ${ }^{\mathrm{e}}$, elle revient sur la genèse et les fondements de la législation moderne en matière funéraire. Se nourrissant des observations et expérimentations menées par des médecins et des chimistes, les débats sur la salubrité des cimetières aux lendemains de la Révolution aboutissent à la définition d'un «bon » cimetière - celui qui décompose rapidement les corps sans rejeter de miasmes cadavériques dans l'atmosphère - et au décret sur les sépultures du 23 prairial an XII qui pose un certain nombre de règles en matière d'implantation des terrains, de profondeur des fosses, de rythme de rotation, etc. Celles-ci seront affinées dans les décennies suivantes. 
7 Le cimetière est donc conçu, selon Anne Carol, "comme machine à consumer les corps » (p.90), où la surface du terrain est déterminée par sa capacité physico-chimique d'absorption, en lien avec le «débit de mortalité ». Elle souligne d'autres indices qui confirment cette analogie, comme le déplacement du thème, dans les rapports annuels des conseils d'hygiène et de salubrité publique, de la rubrique "salubrité publique » à la rubrique "établissement de première classe ": les cimetières sont alors clairement apparentés aux industries les plus dangereuses, comme les chantiers d'équarrissage et les usines métallurgiques. Lorsque cette «machine à consumer » s'enraye au milieu du XIX ${ }^{e}$ siècle - le cimetière étant davantage vécu par ses usagers comme un lieu de conservation, plutôt que comme un lieu de transformation et de destruction - les nouvelles propositions qui voient le jour pour surmonter la surpopulation cimétériale se nourrissent encore du modèle industriel: contribution du chemin de fer au transport des corps, promotion de la crémation, introduction du frigorifique à la morgue, etc.

8 Se penchant ensuite sur les réactions des contemporains, elle rend compte de leurs résistances à cette entreprise de réification des corps et de leurs modes d'appropriation du cimetière, en décalage avec les objectifs de ses concepteurs. La rationalisation industrielle parvenue à s'appliquer dans tous les secteurs de production ne parvient donc pas à s'imposer dans celui du traitement des cadavres. La modernité funéraire, qui se traduit par de nouvelles sensibilités face au corps-mort, semble ainsi entrer en conflit avec la modernité machinique. Il faudra à celle-ci se parer d'habillages ornementaux et rituels pour concilier la consumation des corps et la préservation du souvenir.

9 Ces pistes de recherche présentées par Anne Carol, qui confèrent au cimetière le statut de machines, éclairent d'un angle nouveau la définition des rapports entre corps et machines. La contribution de Nicolas Hatzfeld fait écho à ces tentatives de redéfinition. Si les relations entre ouvriers et machines ont déjà été largement étudiées, il propose, à l'appui des textes de Gilbert Simondon ${ }^{4}$ sur le mode d'existence des objets techniques, de ne pas s'intéresser aux rapports qu'entretiennent les ouvriers à la machine comme outil mécanisé, mais à la machine comme usine, dans son fonctionnement global. Ce pas de côté permet de s'intéresser davantage à l'évolution des organisations industrielles et à leurs impacts sur la santé au travail qu'aux rapports qu'entretient l'ouvrier avec sa machine et évite de s'enfermer dans la perspective jusqu'alors privilégiée par les ingénieurs et les sociologues, comme Alain Touraine, d'une substitution des machines aux hommes.

10 "Comment prendre historiquement la mesure de la souffrance quotidienne » (p. 151) $\mathrm{du}$ travailleur sur une chaîne automobile, s'interroge Alain P. Michel dans une contribution remarquée pour sa volonté "d'inventer de nouvelles sources historiques " ? S'il est aisé de constater des écarts entre les représentations du travail et les pratiques, le travail réel demeure en effet difficile à documenter car « la plupart des documents sont muets et l'historien perplexe». À partir d'une étude de cas l'atelier C5 des usines Renault de Billancourt, en 1922, considéré comme un lieu privilégié d'observation du corps au travail - il témoigne de l'apport des technologies d'image numérique, et notamment de la possibilité de reconstituer en 3D un espace, des gestes et leurs rythmes à partir d'un corpus de documents visuels, croisé avec des sources écrites. L'approche est particulièrement féconde à condition de ne pas ignorer les conditions de production des images et des fonds documentaires et de considérer 
cette "plateforme interactive d'interprétation" (p. 164) comme un instrument de la recherche historique et non comme un aboutissement.

11 Un ouvrage foisonnant, redisons-le, où l'on croise également des études inscrites dans un registre épistémologique (la naissance de la psychologie scientifique par exemple), d'autres qui rendent compte des mécanismes socio-historiques d'appropriation sociale de machines (comme le vélocipède et l'avion qui ont révolutionné le rapport au temps et à l'espace), d'autres encore narrant l'histoire d'un objet, d'une machine, etc. On retiendra surtout ce qui semble constituer l'apport essentiel de ce recueil collectif : la remise en interrogation de ce qui fait machine et de ce qui fait corps dans les relations ambivalentes qui les lient et, partant, une contribution aux recherches sur les transformations du travail et de l'emploi au regard des dommages au corps.

\section{NOTES}

1. Friedmann G. (1945), «L'Homme et le milieu naturel : panorama du nouveau milieu », Annales d'histoire sociale, vol. $8 \mathrm{n}^{\circ}$ 2, p. 116 .

2. La «nouvelle histoire » représente un courant historiographique apparu en France à la fin des années 1970, porté notamment par Jacques le Goff, Emmanuel Le Roy Ladurie, Michel de Certeau, qui élargit le territoire de recherche de l'historien aux mentalités, aux émotions, aux sensibilités, à partir de tous types de matériaux et en s'ouvrant aux méthodes et outils d'autres disciplines comme la sociologie, l'anthropologie, la démographie.

3. Au sens de frontière ici ( $c f$. infra).

4. Philosophe français (1924-1989), auteur ([1958] 1989), Du mode d'existence des objets techniques, Paris, Aubier.

\section{AUTEURS}

\section{ANNE MARCHAND}

Université d'Évry Val d'Essonne, LHEST ; Université d'Aix-Marseille, LAMES 